\title{
A fast alternative to the galvanostatic intermittent titration tech- nique
}

\author{
Yu-Chuan Chien ${ }^{\mathrm{a}}$, Haidong Liu ${ }^{\mathrm{a}}$, Ashok S. Menon ${ }^{\mathrm{a}}$, William R. Brant ${ }^{\mathrm{a}}$, Daniel Brandell ${ }^{\mathrm{a}, *}$, Mat- \\ thew J. Lacey ${ }^{b, *}$ \\ ${ }^{a}$ Department of Chemistry—Ångström Laboratory, Uppsala University, Box 538, Lägerhyddsvägen 1, 75121 Uppsala, \\ Sweden \\ ${ }^{\mathrm{b}}$ Scania CV AB, 15187 Södertälje, Sweden
}

\begin{abstract}
The galvanostatic intermittent titration technique (GITT) has been regarded as the go-to method for determining the diffusion coefficients of Li-ions in insertion electrode materials at various states of charge (SoC). However, the method is notoriously time-consuming. In this work, the intermittent current interruption (ICI) method, which has previously been employed to investigate the progression of internal resistances in Li-ion cells, is demonstrated to provide comparably accurate measurements of diffusion coefficients with a drastically reduced experimental time. Theoretically, it is first derived from Fick's laws that the ICI method renders essentially the same information as GITT. Experimentally, both GITT and ICI are then compared side-by-side in a three-electrode half-cell of $\mathrm{LiNi}_{0.8} \mathrm{Mn}_{0.1} \mathrm{Co}_{0.1} \mathrm{O}_{2}(\mathrm{NMC} 811)$. It is shown that the results from both methods match where the assumption of semi-infinite diffusion applies. Moreover, the benefit of the comparatively non-disruptive ICI method to operando characterization methods is demonstrated via correlation of changes in the continuously monitored diffusion coefficient of $\mathrm{Li}^{+}$in NMC811 to structural changes in the material by operando Xray diffraction (XRD).
\end{abstract}

\section{Introduction}

As the demand for electrochemical energy storage surges, the research, development and application of new systems require comprehensive understanding of the electrochemical properties at an ever-increasing pace. A critical parameter for the community, from materials chemists to application engineers, is the diffusion coefficient of the redoxactive species; i.e., $\mathrm{Li}^{+}$, in the case of Li-ion batteries. The GITT has been the most widely applied method for deriving the diffusion coefficient from electrochemical measurements. Derived from Fick's second law, GITT was first demonstrated in 1977 for a bulk $\mathrm{Li}_{3} \mathrm{Sb}$ electrode. ${ }^{1}$ The technique consists of two repeating steps. First, a constant current is applied for a duration where the assumption of semi-infinite diffusion holds. Second, the current is switched off until the voltage becomes invariant, which indicates that equilibrium is reached. Through the analysis of the electrode potential measured during the current pulse and the change in the equilibrium potential, GITT renders the chemical diffusion coefficient of the chargecarrying ions. (The difference between chemical and tracer diffusion coefficients is elaborated in the original GITT manuscript. ${ }^{1}$ For simplicity, the former will be referred to as diffusion coefficient in the following text.) Later, the technique was applied to porous composite electrodes of Li-ion-insertion materials, ${ }^{2}$ which is the format of the majority of electrodes in state-of-the-art Li-ion batteries. Although practical issues, such as nonuniform current distri- bution, can occur due to the geometry of composite electrodes, ${ }^{3}$ the technique serves as a powerful tool for the determination of diffusion coefficients if proper experimental parameters are chosen, ${ }^{4,5}$ e.g. appropriate current and duration of the current pulses.

However, the time required to perform a GITT measurement remains one major drawback. In order to reach the equilibrium condition, the test cell has to be relaxed substantially longer than the time spent on applying current. ${ }^{5}$ This results in an experiment that can be anywhere from 8 to 100 times longer than a typical galvanostatic test cycle. ${ }^{4,5}$ Although the test may be accelerated by increasing the duration of current pulses and selecting only the initial datapoints for the analysis, ${ }^{6}$ this reduces the number of measurements of diffusion coefficient. In addition, such a test protocol makes it difficult to couple GITT with simultaneous materials characterization, e.g. diffraction or spectroscopy, which can provide valuable structural and/or chemical information at the moment where the process under investigation takes place. In other words, the length of time needed for a typical GITT experiment essentially precludes its use in operando methods, and hence the coupling of operando methods with diffusion coefficient measurements.

In this work, an efficient and non-disruptive alternative to the GITT is proposed: the intermittent current interruption (ICI) method. Our group first developed the ICI method for characterizing the porous carbon electrodes in lithium-sulfur batteries, ${ }^{7-9}$ and the method has more recently been applied more widely for different battery cell 
chemistries. $^{10-12}$ The method introduces transient current interruptions (usually 1 to 10 seconds) while the cell is under constant-current cycling. By analyzing the potential change during the current pauses, quantities describing the time-independent and time-dependent parts of the resistance can be derived, which are termed internal resistance and diffusion resistance coefficient, respectively. ${ }^{9}$ With the porous electrode model, ${ }^{13}$ it has been shown that the derived diffusion resistance coefficient is proportional to the coefficient of the Warburg element used when fitting electrochemical impedance spectra (EIS). ${ }^{9}$ Since the Warburg element describes both the capacitive behavior in porous electrodes ${ }^{13,14}$ and diffusion processes ${ }^{15-17}$, it is a logical consequence that the ICI method can also characterize diffusion processes in an electrochemical system.

We here demonstrate mathematically that the ICI method can render the diffusion coefficient based on Fick's second law and requires much less experimental time than GITT. The theoretical derivations of GITT and ICI are elaborated and compared. Three-electrode cells of NMC811 are then tested following a protocol that enables the direct comparison of GITT, ICI and EIS. Finally, the combination of operando XRD and the ICI method is demonstrated to directly correlate the structural evolution to the Li-ion mobility. This example manifests not only the efficiency of the ICI method in probing the transport properties, but also its compatibility with operando techniques. Moreover, it also indicates the potential of the ICI method as a tool for state of heath estimation of $\mathrm{Li}$-ion batteries.

\section{Theory and procedure}

\subsection{Derivation of GITT}

The derivation of GITT starts from the solution to Fick's second law in one-dimension ${ }^{1}$ :

$$
\frac{\partial C(x, t)}{\partial t}=D \frac{\partial^{2} C(x, t)}{\partial x^{2}}
$$

where $C$ is the concentration of the diffusing species, $x$ is the position, $t$ is time and $D$ is the diffusion coefficient. The boundary conditions with an applied current $i(t)$ and an initial concentration $C_{o}$ are as follows:

$$
\left\{\begin{array}{c}
-D \frac{\partial C}{\partial x}=\frac{i(t)}{n F A} \\
C(x, 0)=C_{0}
\end{array}\right.
$$

where $n$ is the charge number of the diffusing species (which is $\mathbf{1}$ for Li ion), $F$ is the Faraday constant and $A$ is the area of the surface where the diffusing ions enter. For GITT, a constant current $I$ is applied.

$$
i(t)=I
$$

With the above, $C(x, t)$ can be solved for planar diffusion ${ }^{1}$ or spherical diffusion if transformed to the spherical coordinates, ${ }^{4,18-21}$ which is detailed in Equation $\mathrm{S}_{3}$ in the Supporting Information (SI). In either case, when $t \ll L^{2} / D$, where $L$ is the diffusion length or particle radius in the spherical case, semi-infinite diffusion can be assumed and the concentration at the surface can be expressed as:

$$
C(0, t)=C_{0}-\frac{2 I \sqrt{t}}{F A \sqrt{D \pi}}
$$

$$
\frac{d C(0, t)}{d \sqrt{t}}=-\frac{2 I}{F A \sqrt{D \pi}}
$$

Supposing that the change in concentration is small and thus linear to the change in the potential $E$ but in the opposite direction (i.e., the electrode potential increases with decreasing Li-ion concentration), the above expression can be expanded to:

$$
\frac{d E}{d \sqrt{t}}=\frac{2 I}{F A \sqrt{D \pi}} \frac{d E}{d C(0, t)}
$$

With both derivatives of $E$ extracted from experimental data, which will be elaborated in section 2.3, the diffusion coefficient can be calculated by reorganizing Equation 6 .

$$
D=\frac{4}{\pi}\left(\frac{I}{F A} \frac{\frac{d E}{d C(0, t)}}{\frac{d E}{d \sqrt{t}}}\right)^{2}
$$

\subsection{Derivation of the $\mathrm{ICI}$ method}

Instead of analyzing the potential change from open circuit to a constant current load, the ICI method utilizes the opposite case where the current is switched to zero from a constant current load. Considering a constant current $I$ being applied from $t=0$ and switched off at $t=\tau_{1}>0$, Equation 3 is changed to:

$$
i(t)=I-I H(t)
$$

where $H$ is the Heaviside function, defined as:

$$
H(t)= \begin{cases}0, & t<\tau_{1} \\ 1, & t \geq \tau_{1}\end{cases}
$$

By inserting Equation 8 into Equation 2 as the boundary conditions, Equation 1 can now be solved as the following by the Zero-Shift Theorem. ${ }^{15}$

$$
C(0, t)=C_{0}+F(t)-H(t) F\left(t-\tau_{1}\right)
$$

where $C_{o}+F(t)$ is the full solution of Equation 1 when $i(t)$ $=I$, shown in Equation $S_{3} . F\left(t-\tau_{1}\right)$ can be approximated by the semi-infinite diffusion case $\left(t<<L^{2} / D\right)$ since the ICI method only analyzes the potential change in a short period $\Delta \mathrm{t}$ after the current is switched off, which means $\Delta t=$ $t-\tau_{1}<<L^{2} / D$. Thus, when $t \geq \tau_{1}$, Equation 10 can be written as:

$$
C(0, t)=C_{0}-F(t)+\frac{2 I \sqrt{t-\tau_{1}}}{F A \sqrt{D \pi}}
$$

So, the surface concentration at $t=\tau_{1}+\Delta t$ is:

$$
C\left(0, \tau_{1}+\Delta t\right)=C_{0}-F\left(\tau_{1}+\Delta t\right)+\frac{2 I \sqrt{\Delta t}}{F A \sqrt{D \pi}}
$$

Assuming that $\tau_{1}$ is so much larger than $\Delta t$ that $F\left(\tau_{1}+\Delta t\right)$ $\approx F\left(\tau_{1}\right)$ and thus independent of $\Delta t$, which is a criterion for the ICI method and is discussed in the SI, the following is obtained:

$$
\frac{d C(0, t)}{d \sqrt{\Delta t}}=\frac{2 I}{F A \sqrt{D \pi}}
$$

This expression is similar to Equation 5 and if the change in concentration is small, it can be expanded to:

$$
-\frac{d E}{d \sqrt{\Delta t}}=\frac{2 I}{F A \sqrt{D \pi}} \frac{d E}{d C(0, t)}
$$


In the analysis of the ICI method, ${ }^{8,9}$ which will be elaborated in section 2.4, $d E / d \sqrt{\Delta t}$ is readily obtained. How $d E / d C(o, t)$ can be obtained with the ICI method will be discussed in section 2.4. With both $d E / d \sqrt{t}$ and $d E / d C(o, t)$ obtainable from the ICI method, the diffusion coefficient can then be calculated from Equation 7.

\subsection{Experimental execution of GITT}

To employ Equation 7 in a conventional GITT measurement, the two derivatives of $E$ ( $d E / d C$ and $d E / d \sqrt{\Delta t}$ ) have to be determined. For $d E / d C$, the change in concentration of the charge carrier is not directly measured but can be calculated under constant current.

$$
d C=\frac{I d t_{I}}{F V}
$$

where $V$ is the volume of the electrode and $d t_{I}$ is the duration of the applied current. Assuming that $d E / d C$ changes relatively slowly and thus can be interpolated by $\Delta E / \Delta C{ }^{1,6}$ Equation 7 can be rewritten as follows.

$$
D=\frac{4}{\pi}\left(\frac{V}{A} \frac{\frac{\Delta E_{O C}}{\Delta t_{I}}}{\frac{d E}{d \sqrt{t}}}\right)^{2}
$$

where $\Delta E_{O C}$ is the change in the open-circuit potential (OCP) from the rests before and after the current pulse, and $\Delta t_{I}$ is the period of the current pulse (denoted as $\tau$ in the original paper ${ }^{1}$ ). It is worth noting that Equation 15 applies to the concentration of the entire electrode. In order to relate the measured electrode potential, which reflects the surface concentration, to the bulk concentration, the measurement should ideally be done when the concentration is uniform throughout the electrode. This is indicated by the fully relaxed electrode potential, i.e. $d E / d t=o$, also known as the open-circuit condition. In practice, it takes long time to achieve equilibrium in the electrode, which is the reason for the substantial time consumption of GITT. ${ }^{5}$

$d E / d \sqrt{t}$ is the slope on the plot of $E$ against $\sqrt{t}$. In the original GITT paper, ${ }^{1}$ Equation 16 is further reduced by assuming that $E$ is linear to $\sqrt{t}$ during the whole current pulse. However, this assumption is less likely to hold for electrode particles in a composite electrode since the duration for the semi-infinite diffusion condition, $t<<L^{2} / D$, is reduced by the shorter $L$, compared to the bulk electrode used in the original paper. Two solutions to solve the issue are: 1 ) selecting only the data lying in the linear region on the $E-\sqrt{t}$ plot or 2) fitting the data with the full solution (Equation $\mathrm{S}_{3}$ ) to Fick's second law (method $\mathrm{P}_{3}$ and $\mathrm{P}_{5}$ in the reference, respectively). ${ }^{4}$ In this work, we will proceed with the first solution and examine the effect of data selection on the GITT analysis.

\subsection{Experimental execution of the $\mathrm{ICI}$ method}

The change in the potential after the current has been switched off $(\Delta t=0)$ can be expressed as the following.

$$
\Delta E(\Delta t)=E(\Delta t)-E_{I}=-I R-I k \sqrt{\Delta t}
$$

where $E_{I}$ is the potential right before the current is switched off, and $R$ and $k$ are termed internal resistance and diffusion resistance coefficient, respectively. ${ }^{9} R$ and $k$ are acquired through the linear regression of $\Delta E$ against $\sqrt{\Delta t}$. For repetitive current pauses, the regression can be automated by a script in a common programing languages; $\mathrm{R}^{22}$ has been used in this work.

Comparing Equation 14 and 17, it can be observed that

$$
I k=-\frac{d E}{d \sqrt{\Delta t}}=\frac{2 I}{F A \sqrt{D \pi}} \frac{d E}{d C(0, t)}
$$

which can be reorganized into the form of Equation 16 with Equation 15.

$$
D=\frac{4}{\pi}\left(\frac{V}{A} \frac{\frac{d E_{O C}}{d t_{I}}}{I k}\right)^{2}
$$

Without the relaxation step, $d E_{O C} / d t_{I}$, can be 1) obtained from existing OCP data of the electrode material or 2) approximated by the slope of iR-corrected pseudo-OCP. The iR-drop and pseudo-OCP, referring to the measured potential under low constant current, ${ }^{5}$ are readily available in the ICI analysis. From two neighboring current interruptions, the change in OCP can be approximated by the change in $E(\Delta t=0)$, which is the potential right before the current pause subtracting the iR-drop, as shown in Equation 17. The validity of using $\Delta[E(\Delta t=0)] / \Delta t_{I}$ as $d E_{O C} / d t_{I}$ will be examined in Section 3.

\subsection{Experimental procedure}

Two three-electrode half-cells, termed cells 1 and 2, were made for the validation of the theoretical derivation. The working electrode $\left(\varnothing_{13} \mathrm{~mm}\right)$ was a tape-cast composite electrode consisting of $90 \mathrm{wt} \%$ NMC811 powder (Customcells Itzehoe $\mathrm{GmbH}$ ), $5 \mathrm{wt} \%$ of carbon black (Super C65, Imerys) and $5 \mathrm{wt} \%$ poly(vinylidene difluoride) (PVdF, Solvay), whose fabrication procedure can be found in the previous work. ${ }^{23}$ According to the supplier, the NMC811 particles have a median diameter of $4 \mu \mathrm{m}$ and specific surface area of $1.5 \mathrm{~m}^{2} \mathrm{~g}^{-1}$, determined by the Brunauer-Emmett-Teller (BET) analysis of the nitrogen adsorption isotherm. The specific volume was calculated from the molecular mass and the previously reported (rhombohedral) unit cell parameters obtained through X-ray diffraction (XRD) to be $0.63056 \mathrm{~cm}^{3} \mathrm{~g}^{-1} \cdot{ }^{23}$ The areal loadings of NMC811 were 2.11 and $2.13 \mathrm{mg} \mathrm{cm}^{-2}$ in cells 1 and 2, respectively. Both the counter $\left(\varnothing_{15} \mathrm{~mm}\right)$ and reference electrodes (ring with inner and outer diameters of 16 and $22 \mathrm{~mm}$, respectively) were metallic lithium (Cyprus Foote Mineral, $125 \mu \mathrm{m}$ thick). The reference electrode was placed between the working and counter electrodes with separators (Celgard 2325) on both sides according to a previously reported cell geometry. ${ }^{10}$ The cells were assembled in an Ar-filled glove box with $1 \mathrm{M}$ $\mathrm{LiPF}_{6}$ in a mixture of ethylene carbonate/diethylene carbonate (EC/DEC 1:1 by volume, Solvionic, purity: $99.9 \%$ ) as the electrolyte and sealed in pouch bag material. Assembled cells were rested for 12 hours before 3 pre-cycles at 20 $\mathrm{mA} \mathrm{g}^{-1}$ between 3.0 and $4.3 \mathrm{~V}$. Electrochemical tests were carried out using a Biologic MPG 2.

A slightly modified GITT protocol was designed here to compare the GITT, ICI and EIS at the same SoC, which is schematically shown in Figure 1. A constant current of 20 
$\mathrm{mA} \mathrm{g}^{-1}$ (corresponding to $\mathrm{C} / 1 \mathrm{o}$, where $\mathrm{C}$ is here defined as $200 \mathrm{~mA} \mathrm{~g}^{-1}$ for NMC811) was applied for 10 minutes, which was followed by a 1-hour rest. During the first minute of the rest, potential was recorded every o.1 second for the ICI analysis. After the rest, an EIS measurement was performed from $20 \mathrm{kHz}$ to $10 \mathrm{mHz}$ with an amplitude of $10 \mathrm{mV}$. Another 10-minute rest followed the EIS measurement before the next current pulse. The modified GITT protocol was applied between 3.0 and $4.3 \mathrm{~V}$ for two cycles. In the second discharge, the cutoff was lowered to 2.0 and $2.5 \mathrm{~V}$ for cell 1 and 2, respectively. For both GITT and ICI analysis, the electrode volume and area were approximated by the volume and surface area of the NMC particles stated above. The impedance spectra, where a Warburg element is present, were fitted to the equivalent circuit model in Figure S2 by a modified Levenberg-Marquardt algorithm provided by the "minpack.lm" package in the R-programming language. $^{24}$

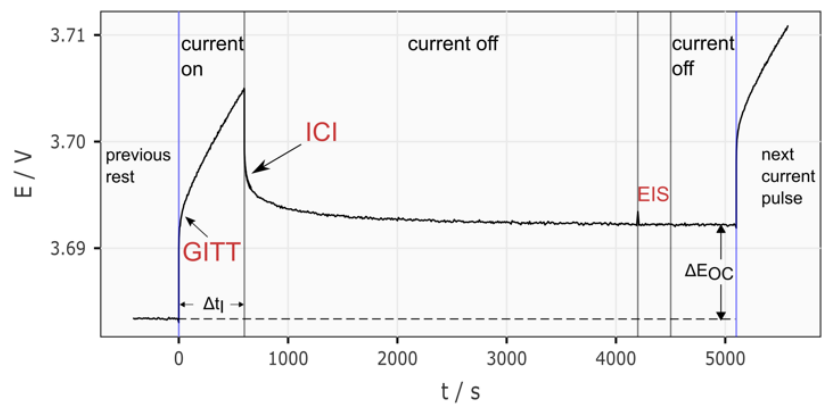

Figure 1. Electrode potential (E) plotted against time (t) during a current pulse $\left(I=20 \mathrm{~mA} \mathrm{~g}^{-1}\right)$ and a rest period $(\mathrm{I}=0)$ of the modified GITT program used in this work for the comparison of the results from the GITT, ICI method and EIS.

After the above-described test, the cells went on to be cycled with the standard ICI protocol. Both cells were charged to $4.3 \mathrm{~V}$ and discharged to $3 \mathrm{~V}$ at a constant current of $20 \mathrm{~mA} \mathrm{~g}^{-1}$. A 10-second current interruption every 5 and 15 minutes was introduced to cells 1 and 2, respectively. During the $57^{\text {th }}$ discharge of cell 1 , an operando XRD experiment was performed on the cell as it was charged up to $4.3 \mathrm{~V}$ and subsequently discharged to $3.7 \mathrm{~V}$ at $20 \mathrm{~mA} \mathrm{~g}$ with a 10-second current interruption every 5 minutes, as in the previous cycles. Patterns were recorded by a STOE STADI P diffractometer in transmission setup with monochromatic $\mathrm{Cu}-\mathrm{K}_{\alpha 1}$ radiation every 15 minutes using a Dectris Mythen $21 \mathrm{~K}$ detector setup. Rietveld refinements ${ }^{25,26}$ were performed against the XRD data using the Topas-Academic software (V6). ${ }^{27}$ Further details of the refinements and the results are provided in the SI (Section 5).

Raw data from both the electrochemical and operando XRD experiments, the R-scripts used for the GITT, ICI and EIS analyses and the results of the Rietveld refinements can be accessed via Zenodo. ${ }^{28}$

\section{Results and Discussion}

Since cells 1 and 2 are identical and thus show similar behaviors in both cycles of the modified GITT protocol, the results of cell 1 in first cycle are discussed in detail while the rest is presented in the SI.

As shown in Equations 7, 16 and 19, to derive the diffusion coefficient, two measurements are required: $d E / d \sqrt{t}$ during semi-infinite diffusion and the slope of OCP. Therefore, the following text will first compare the two values obtained by the GITT and ICI method. Then, the diffusion coefficients calculated from the two methods will be presented. The data acquired during the current pulses and the rest periods are analyzed by the GITT and ICI methods, respectively, as indicated in Figure 1. For the GITT, two data selection intervals, 5-40 and 50-150 s, were utilized because they contain the linear region of the $E-\sqrt{t}$ plot above and below $3.7 \mathrm{~V}$, respectively. An example of each case is plotted in Figure S1. For the ICI method, the interval was chosen to be $0.2-5 \mathrm{~s}$ for the same criteria applied on the data during the rest periods.

Figure 2 displays the $k$ values, which are $d E / d \sqrt{t}$ normalized by the current (Equation 18) from GITT and ICI, and the Warburg coefficients $(\sigma)$ multiplied by $\sqrt{8 / \pi}$ from EIS fittings. This linear relationship of $k=\sigma \sqrt{8 / \pi}$ has been demonstrated in previous work. ${ }^{9}$ Above $3.7 \mathrm{~V}$, the $k$ values determined by ICI and GITT with 5-40 s interval are close to each other, which confirms the theoretical derivation in section 2.2 and is corroborated by the EIS results. The GITT results collected from the 50-150 s interval show higher $\mathrm{k}$ values and two local maxima between 3.7 and 4.2 $\mathrm{V}$ while the $k$ values from other three analyses fluctuate less and are more consistent with each other. This is expected from the data beyond the time scale of semi-infinite diffusion, as the surface concentration and thus the potential $E$ then becomes linear with $t$, instead of $\sqrt{t}$, (Equation $\left.S_{5}\right)$, leading to an overestimation of $d E / d \sqrt{t}$.

Below $3.7 \mathrm{~V}$, the linear region on the $E-\sqrt{t}$ plots shifts to 50-150 s, as demonstrated in Figure S1. This means that only the GITT result from the 50-150 s interval properly provides diffusion coefficients below $3.7 \mathrm{~V}$. This phenomenon is also manifested by the expansion of the second semi-circle on impedance spectra (Figure $\mathrm{S}_{4}$ ), which do not show a Warburg element within the frequency range $(20 \mathrm{kHz}-10 \mathrm{mHz}$ ) and thus cannot render accurate $k$ values. The increase in charge transfer resistance represented by the enlarged semi-circle has been reported for NMCi11 $\left(\mathrm{LiNi}_{0.33} \mathrm{Mn}_{0.33} \mathrm{Co}_{0.33} \mathrm{O}_{2}\right)$ at low SoC., ${ }^{2,17}$ In summary, Figure 2 illustrates the consistency of EIS, GITT and ICI method within their respective limitations and the importance of selecting the proper time interval where $d E / d \sqrt{t}$ is linear when conducting the GITT analysis.

The other quantity experimentally determined in these methods used in the calculation of the diffusion coefficient (Equations 16 and 19) is the OCP slope. Figure 3 presents a comparison between the slopes of the OCP obtained from the relaxed potential at the end of the rest period in the GITT protocol and the iR-corrected pseudo-OCP in the ICI analysis. The difference between the values from two methods are minimal above $3.65 \mathrm{~V}$. The deviation at low $\mathrm{SoC}$ is presumably linked to the high resistance discussed above, which interferes with the resistance determination 
of the ICI method. Nevertheless, the good agreement between the slopes of OCP and iR-corrected pseudo-OCP in most SoC intervals indicates that the ICI method alone can deliver the required electrochemical parameters for the calculation of the diffusion coefficient above 3.7 V. By skipping the time-consuming relaxation periods, the ICI method can save around $90 \%$ of the time spent on common GITT protocols, such as the one used in this work.

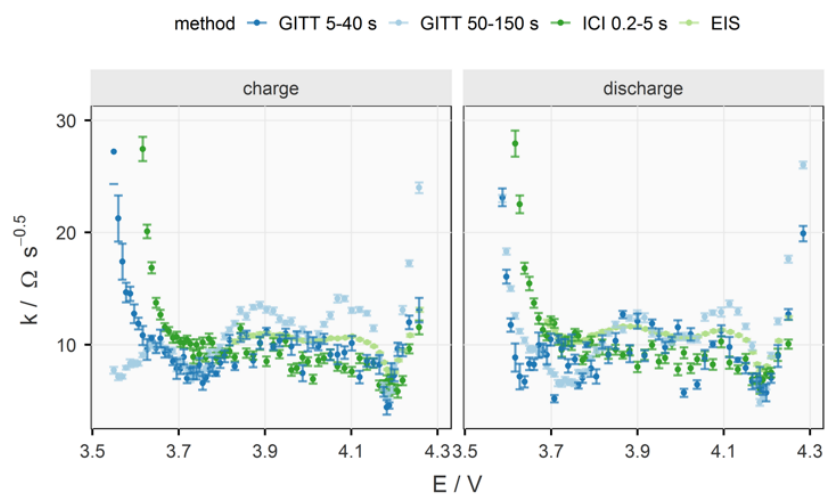

Figure 2. The diffusion resistance coefficient (k) in NMC811(D) in cell 1 at various OCP of the electrode $(\mathrm{E})$ against $\mathrm{Li} / \mathrm{Li}^{+}$derived from the GITT with data selection interval $5-40 \mathrm{~s}$ and 50-150 s, the ICI method and the EIS fitting $(k=\sigma \sqrt{8 / \pi}, \sigma$ : Warburg coefficient). The maximum of the $y$-axis is set to 30 $\Omega \mathrm{s}^{-0.5}$ to show the differences of the data above $3.7 \mathrm{~V}$. Due to a technical issue, the spectra below $3.8 \mathrm{~V}$ in the first charge were not properly collected, but it was solved afterwards.

With both experimental inputs verified, the diffusion coefficients of $\mathrm{Li}^{+}$in NMC811 at various SoC obtained by the GITT and ICI method are exhibited in Figure 4. Overall, the results from the three analyses are close to each other and previously reported $\mathrm{Li}^{+}$diffusion coefficients in NMC811. ${ }^{5,29}$ Above $3.7 \mathrm{~V}$, the match is especially close for the values from the GITT with 5-40 s interval and the ICI method. This is expected since the $d E / d \sqrt{t}$ values derived from both GITT and ICI method are in good agreement in Figure 2 and the slopes of OCP from both methods are basically the same above $3.65 \mathrm{~V}$. Below $3.7 \mathrm{~V}$, differences between three analyses are obvious. In principle, the GITT results from the 50-150 s interval is the most credible one among the three because the interval contains the linear region of the $\mathrm{E}-\sqrt{t}$ plots at these $\mathrm{SoC}$ values. However, the extension of the data selection interval requires the increase in the time limit of semi-infinite diffusion $\left(t<L^{2} / D\right)$. The assumption is satisfied upon discharging due to the much lower diffusion coefficients, but not on charging. This issue will be discussed in details in Section 1 of the SI.

It is mentioned in section 2.5 that the BET-surface area is used as $A$ in Equations 16 and 19, which may differ from the electrochemically active surface area. However, the objective of this work is to compare the GITT and ICI method, both of which are equally affected by this factor.

Other valuable information provided by the ICI method is the internal resistance $(R)$, as shown in Figure $5 . R$ values derived from the iR-drop in the GITT and ICI method are compared with the sum of Ro, R1 and R2 from EIS (Figure
S2). The results from ICI and EIS are almost identical across the whole range of SoC. The GITT with 5-40 s interval yields similar $R$ values during discharge but larger values upon charging. The results from the GITT with 50-150 $\mathrm{s}$ interval are scattered compared to the other methods. Nonetheless, all four methods confirm the high internal resistance below $3.7 \mathrm{~V}$, which changes the linear region of $E$ $\sqrt{t}$ plots, as discussed above. The internal resistance has been reported to be an important indicator for ageing of NMC8 $11^{30}$ and utilized for detecting Li-plating in commercial Li-ion cells. ${ }^{12}$

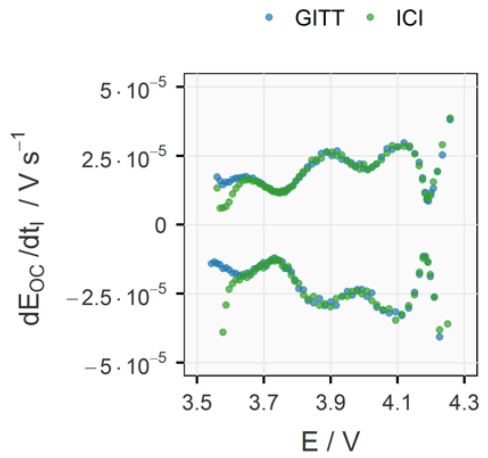

Figure 3. The slope of the OCP $\left(\mathrm{dEOC}_{\mathrm{O}} / \mathrm{dt}_{\mathrm{I}}\right)$ obtained from the relaxed potentials at the end of each rest period (GITT) is compared with the slope of the potential under constant-current load subtracting the iR-drop derived from the ICI method $\left(\Delta[E(\Delta t=o)] / \Delta t_{\text {I, }}\right.$ marked as ICI $)$.

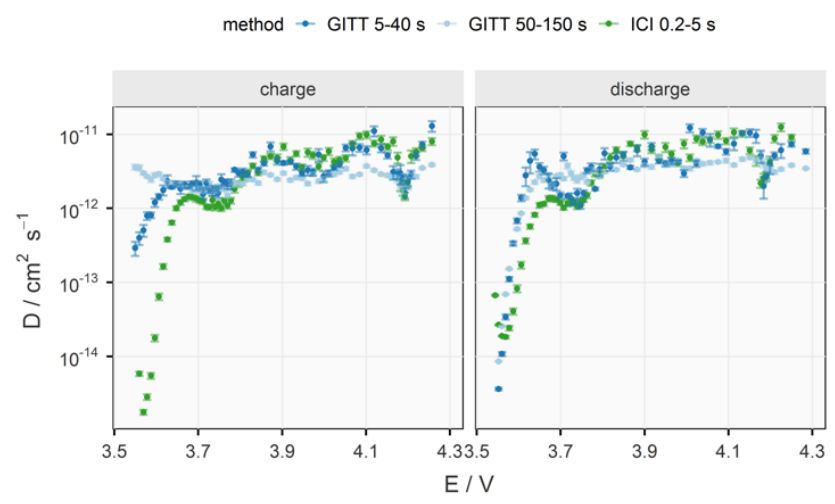

Figure 4. The Li-ion diffusion coefficient in NMC811 (D) in cell 1 at various OCP of the electrode (E) against $\mathrm{Li} / \mathrm{Li}^{+}$derived from the GITT with data selection interval 5-40 s and 50-150 s, the ICI method with the OCP slope from GITT and the pseudo-OCP slope at $20 \mathrm{~mA} \mathrm{~g}^{-1}$.

The convenience of the ICI method is further illustrated by the results in Figure 6, which show the change in Li-ion diffusion coefficient and internal resistance over more than 50 cycles. In the upper two panels, a clear decrease in the Li-ion diffusion coefficient can be observed above $4.2 \mathrm{~V}$. The rate of decrease is higher in the first 10 cycles than in the following cycles. This SoC range, 4.2-4.3 V, corresponds to the drastic shrinkage of the $c$ lattice parameter of the rhombohedral $(R \overline{3} \mathrm{~m})$ unit cell, which has also been reported in several operando XRD studies of NMC811. ${ }^{23,31-33}$ On the other hand, the internal resistance increases more 
uniformly in all SoC above $3.7 \mathrm{~V}$ and the rate is faster in the initial cycles.

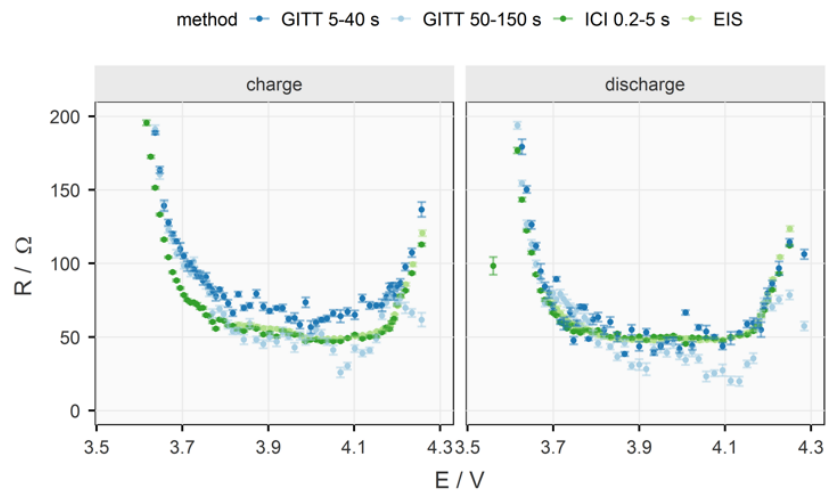

Figure 5. The internal resistance (R) of NMC811 in cell 1 at various OCP of the electrode (E) against $\mathrm{Li} / \mathrm{Li}^{+}$derived from the GITT with data selection interval 5-40 s and 50-150 s, the ICI method and the EIS fitting $\left(\mathrm{Ro}_{0}+\mathrm{R}_{1}+\mathrm{R}_{2}\right)$. The maximum of $\mathrm{y}-$ axis is set to $200 \Omega$ to show the differences of the data above $3.7 \mathrm{~V}$.

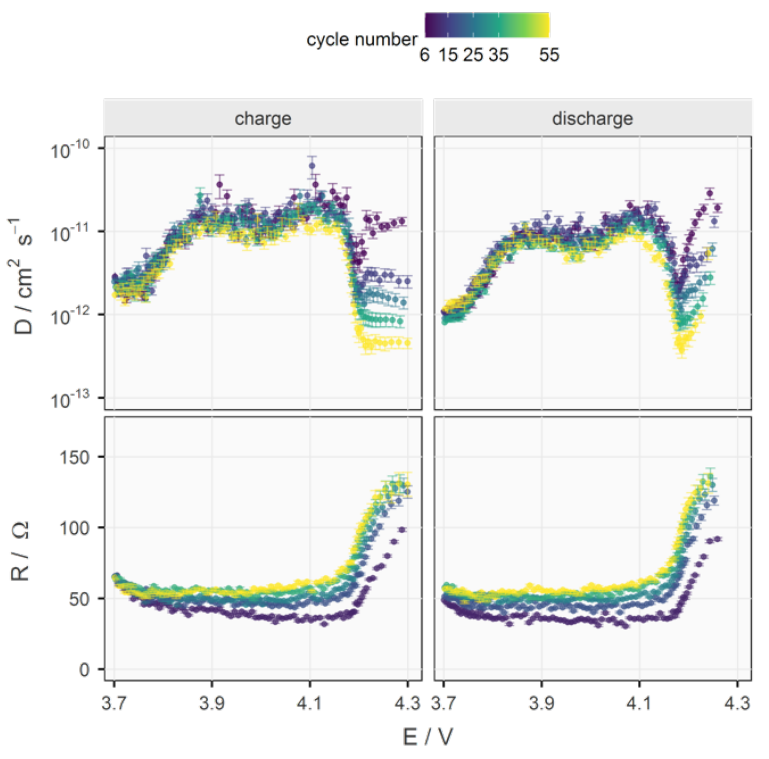

Figure 6. The Li-ion diffusion coefficient in NMC811(D) and internal resistance $(R)$ of cell 1 at various electrode potential (E) against $\mathrm{Li} / \mathrm{Li}^{+}$derived by the ICI method in cycle $6,15,25$, 35 and 55. Note that the electrode potential here is obtained while cycling, not the OCP. Only values with $\mathrm{E} \geq 3.7 \mathrm{~V}$, where the ICI method is applicable as discussed above, are shown.

To further demonstrate the flexibility of the ICI method, an operando XRD experiment coupled with the ICI method was performed on cell 1 after 56 cycles. As depicted in Figure 7 , when the 003 reflection $(\mathrm{R} \overline{3} \mathrm{~m})$ shifts to higher $2 \theta$ values, the diffusion coefficient drops over an order of magnitude and the internal resistance tripled. The shift of the 003 reflection starts at $4.1 \mathrm{~V}$ and accelerates at $4.2 \mathrm{~V}$, which coincides with the start of the increase in internal resistance and the decrease in diffusion coefficient, respec- tively. The reverse can be observed during discharge. In addition, by comparing the patterns taken above $4.2 \mathrm{~V}$ with a previous operando XRD study on the same material in the first cycle ${ }^{23}$ it can be observed that in the degraded cell here, the 003 reflection is composed of two rhombohedral phases with dissimilar c lattice parameters (Figure S16). The exact mechanism for this phase separation is still debated, but most models attribute this to the ramifications of the formation of the degraded rock salt phase. ${ }^{31,34,35} \mathrm{Nev}-$ ertheless, it is shown here that the ICI method can be easily combined with operando XRD and track the diffusion coefficient and internal resistance in real time, which constitutes a valuable method for further studies of the degradation mechanisms of this and other materials.

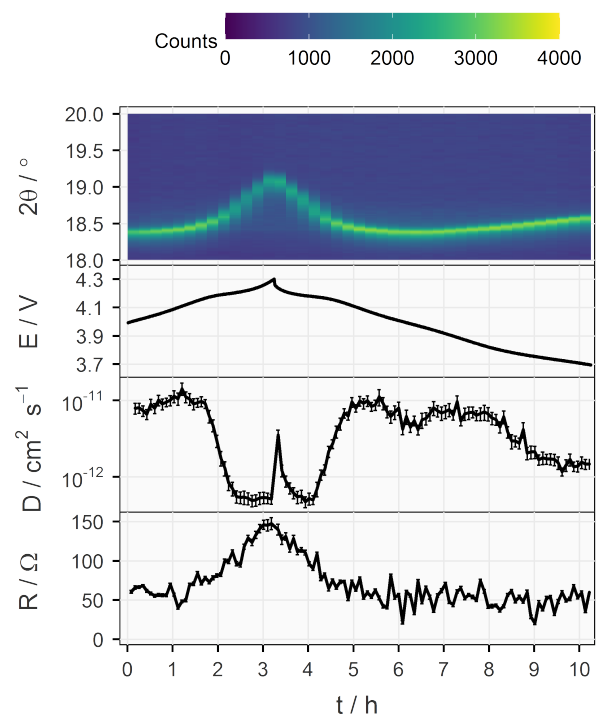

Figure 7. The evolution of the oo3 (first panel) reflections $(\mathrm{Cu}-$ $K \alpha_{1}$ ) as a heat map, electrode potential (E), Li-ion diffusion coefficient in NMC811 (D) and the internal resistance (R) from the combination of operando XRD and the ICI method conducted on cell 1 after 56 galvanostatic cycles between 3.0 and $4.3 \mathrm{~V}$.

\section{Conclusions}

This work establishes the theoretical foundation and experimental validation for the application of the ICI method as an efficient alternative to GITT. Provided that 1 ) the diffusion process under investigation exhibits the semi-infinite diffusion behavior within the maximum time allowed for the current interruption and that 2) the pseudo-OCP slope is a good approximation of the true OCP slope, the ICI method can yield the diffusion coefficient with a much shorter experimental time. In the case of our validation experiment with NMC811, more than $90 \%$ of the time required for a typical GITT experiment can be saved. Moreover, the internal resistance and diffusion resistance coefficient (or equivalently, the Warburg coefficient) determined by the ICI method are also verified by EIS for NMC811. The efficient determination of diffusivity and resistance unlocks new applications which GITT and EIS are 
deemed too time- or resource-consuming, such as online cell parameterization for adaptive charging protocol and simultaneous observation of operando spectro-/diffractometry and electrochemical impedance/resistance. When exemplified by a combination of operando XRD and the ICI method, the rapid decrease of the Li-ion diffusion coefficient above $4.2 \mathrm{~V}$ over cycling could be correlated to the increasing irreversibility of the contraction and elongation of the $c$ lattice parameter of the NMC structure. To our knowledge, it is the first report of concurrent characterization of the crystal structure and the $\mathrm{Li}^{+}$diffusion coefficient.

\section{ASSOCIATED CONTENT}

Supporting information available: The material includes the following sections.

1. Full solution to Fick's Second Law

2. Limit of the current interruption time $(\Delta t)$ of an ICI measurement

3. Equivalent circuit model and selected impedance spectra 4. Data from both cells in both cycles

5. Analysis of the operando X-ray diffraction (XRD) results This material is available free of charge via the Internet at http://pubs.acs.org.

\section{AUTHOR INFORMATION}

\section{Corresponding Author}

* Daniel Brandell

daniel.brandell@kemi.uu.se

* Matthew J. Lacey

matthew.lacey@scania.com

\section{Author Contributions}

The manuscript was written through contributions of all authors.

\section{Funding Sources}

Any funds used to support the research of the manuscript should be placed here (per journal style).

\section{Notes}

The authors declare no competing financial interest. Raw data from electrochemical experiments, the scripts for GITT, ICI and EIS analysis, data from the operando XRD experiment and associated plots, are available free of charge at https://doi.org/10.5281/zenodo.4964674

\section{ACKNOWLEDGMENT}

The authors acknowledge the financial support from the STandUP for Energy consortium. A. S. M. is grateful to the Swedish Foundation for Strategic Research (SSF) within the Swedish National Graduate School in Neutron Scattering (SwedNess) for funding. H. L., W. R. B. and M. J. L. are grateful to Swedish Energy Agency (Energimyndigheten) for funding through projects: 51813-1, 48678-1 and 45538-1, respectively.

\section{REFERENCES}

(1) Weppner, W.; Huggin, R. A. Determination of the Kinetic Parameters of Mixed-Conducting Electrodes and Application to the System $\mathrm{Li}_{3} \mathrm{Sb}$. J. Electrochem. Soc. 1977, 124, 1569. https://doi.org/10.1149/1.2133112.

(2) Shaju, K. M.; Subba Rao, G. V.; Chowdari, B. V. R. Influence of Li-Ion Kinetics in the Cathodic Performance of Layered
$\mathrm{Li}\left(\mathrm{Ni}_{1 / 3} \mathrm{Co}_{1 / 3} \mathrm{Mn}_{1 / 3}\right) \mathrm{O}_{2}$. J. Electrochem. Soc. 2004, 151, A1324. https://doi.org/10.1149/1.1775218.

Dees, D. W.; Kawauchi, S.; Abraham, D. P.; Prakash, J. Analysis of the Galvanostatic Intermittent Titration Technique (GITT) as Applied to a Lithium-Ion Porous Electrode. J. Power Sources 2009, 189, 263-268. https://doi.org/10.1016/j.jpowsour.2008.09.045.

Nickol, A.; Schied, T.; Heubner, C.; Schneider, M.; Michaelis, A.; Bobeth, M.; Cuniberti, G. GITT Analysis of Lithium Insertion Cathodes for Determining the Lithium Diffusion Coefficient at Low Temperature: Challenges and Pitfalls. J. Electrochem. Soc. 2020, 167, 090546. https://doi.org/10.1149/1945-7111/ab9404.

Chen, C.-H.; Brosa Planella, F.; O'Regan, K.; Gastol, D.; Widanage, W. D.; Kendrick, E. Development of Experimental Techniques for Parameterization of MultiScale Lithium-Ion Battery Models. J. Electrochem. Soc. 2020, 167, o80534. https://doi.org/10.1149/1945-7111/ab9050.

Wen, C. J.; Boukamp, B. A.; Huggins, R. A.; Weppner, W. Thermodynamic and Mass Transport Properties of "LiAl." J. $\begin{array}{lllll}\text { Electrochem. } & \text { Soc. } & 1979, & 126, & 2258 .\end{array}$ https://doi.org/10.1149/1.2128939.

(7) Lacey, M. J.; Edström, K.; Brandell, D. Visualising the Problems with Balancing Lithium-Sulfur Batteries by "Mapping" Internal Resistance. Chem. Commun. 2015, 51, 16502-16505. https://doi.org/10.1039/C5CCo7167D.

Lacey, M. J. Influence of the Electrolyte on the Internal Resistance of Lithium-Sulfur Batteries Studied with an Intermittent Current Interruption Method. ChemElectroChem 2017, 4, 1997-2004. https://doi.org/10.1002/celc.201700129.

Chien, Y.-C.; Menon, A. S.; Brant, W. R.; Brandell, D.; Lacey, M. J. Simultaneous Monitoring of Crystalline Active Materials and Resistance Evolution in Lithium-Sulfur Batteries. J. Am. Chem. Soc. 2020, 142, 1449-1456. https://doi.org/10.1021/jacs.9b115oo.

(10) Aktekin, B.; Lacey, M. J.; Nordh, T.; Younesi, R.; Tengstedt, C.; Zipprich, W.; Brandell, D.; Edström, K. Understanding the Capacity Loss in $\mathrm{LiNi}_{0.5} \mathrm{Mn}_{1.5} \mathrm{O}_{4}-\mathrm{Li}_{4} \mathrm{Ti}_{5} \mathrm{O}_{12}$ Lithium-Ion Cells at Ambient and Elevated Temperatures. J. Phys. Chem. C 2018, 122, 11234-11248. https://doi.org/10.1021/acs.jpcc.8bo2204.

(11) Hernández, G.; Naylor, A. J.; Chien, Y.-C.; Brandell, D.; Mindemark, J.; Edström, K. Elimination of Fluorination: The Influence of Fluorine-Free Electrolytes on the Performance of $\mathrm{LiNi}_{1 / 3} \mathrm{Mn}_{1 / 3} \mathrm{Co}_{1 / 3} \mathrm{O}_{2} /$ Silicon-Graphite Li-Ion Battery Cells. ACS Sustain. Chem. Eng. 2020, 8, 10041-10052. https://doi.org/10.1021/acssuschemeng.oco1733.

(12) Koleti, U. R.; Dinh, T. Q.; Marco, J. A New On-Line Method for Lithium Plating Detection in Lithium-Ion Batteries. J. Power Sources 2020, 451, 227798. https://doi.org/10.1016/j.jpowsour.2020.227798.

(13) de Levie, R. On Porous Electrodes in Electrolyte Solutions. Electrochim. Acta $1963, \quad 8, \quad 751-780$. https://doi.org/10.1016/o013-4686(63)80042-o.

(14) Lasia, A. Impedance of Porous Electrodes. J. Electroanal. Chem. 1995, 397, 27-33. https://doi.org/10.1016/o0220728(95)04177-5.

(15) Bard, A. J.; Faulkner, L. R. Electrochemical Methods: Fundamentals and Applications, 2nd ed.; Wiley, 2000.

(16) Lasia, A. Electrochemical Impedance Spectroscopy and Its Applications; Springer New York: New York, NY, 2014. https://doi.org/10.1007/978-1-4614-8933-7.

(17) Charbonneau, V.; Lasia, A.; Brisard, G. Impedance Studies of $\mathrm{Li}+$ Diffusion in Nickel Manganese Cobalt Oxide (NMC) during Charge/Discharge Cycles. J. Electroanal. Chem. 2o2o, 875, 113944. https://doi.org/10.1016/j.jelechem.2020.113944.

(18) Carslaw, H. S.; Jaeger, J. C. Conduction of Heat in Solids, 2nd ed.; Oxford science publications; Clarendon: Oxford, 1986. 
(19) Liu, S. An Analytical Solution to $\mathrm{Li} / \mathrm{Li}+$ Insertion into a Porous Electrode. Solid State Ionics 2006, 177, 53-58. https://doi.org/10.1016/j.ssi.2005.09.053.

(20) Subramanian, V. R.; White, R. E. New Separation of Variables Method for Composite Electrodes with Galvanostatic Boundary Conditions. J. Power Sources 2001, 96, 385-395. https://doi.org/10.1016/So378-7753(oo)oo656-X.

(21) Subramanian, V. R.; Ritter, J. A.; White, R. E. Approximate Solutions for Galvanostatic Discharge of Spherical Particles I. Constant Diffusion Coefficient. J. Electrochem. Soc. 2001, 148, E444. https://doi.org/10.1149/1.1409397.

(22) R Core Team. R: A Language and Environment for Statistical Computing https://www.r-project.org/.

(23) Liu, H.; Naylor, A. J.; Menon, A. S.; Brant, W. R.; Edström, K.; Younesi, R. Understanding the Roles of Tris(Trimethylsilyl) Phosphite (TMSPi) in $\mathrm{LiNi}_{0.8} \mathrm{Mno.1Coo.1 \textrm {O } _ { 2 }}$ (NMC811)/Silicon-Graphite (Si-Gr) Lithium-Ion Batteries. Adv. Mater. Interfaces 2020, 7, 2000277. https://doi.org/10.1002/admi.202000277.

(24) Elzhov, T. V.; Mullen, K. M.; Spiess, A.-N.; Bolker, B. Minpack.Lm: $\mathrm{R}$ Interface to the Levenberg-Marquardt Nonlinear Least-Squares Algorithm Found in MINPACK, Plus Support for Bounds. 2016.

(25) Rietveld, H. M. Line Profiles of Neutron Powder-Diffraction Peaks for Structure Refinement. Acta Crystallogr. 1967, 22, 151-152. https://doi.org/10.1107/s0365110x67000234.

(26) Rietveld, H. M. A Profile Refinement Method for Nuclear and Magnetic Structures. J. Appl. Crystallogr. 1969, 2, 65-71. https://doi.org/10.1107/Soo21889869006558.

(27) Coelho, A. A. TOPAS and TOPAS-Academic: An Optimization Program Integrating Computer Algebra and Crystallographic Objects Written in C++: An. J. Appl. Crystallogr. 2018, 210-218. https://doi.org/10.1107/S1600576718000183.

(28) Chien, Y.-C.; Liu, H.; Menon, A. S.; Brant, W. R.; Brandell, D.; Lacey, M. J. Supporting Data for "A Fast Alternative to the Galvanostatic Intermittent Titration Technique.” 2021. https://doi.org/10.5281/zenodo.4964674.
(29) Hu, J.; Wu, B.; Cao, X.; Bi, Y.; Chae, S.; Niu, C.; Xiao, B.; Tao J.; Zhang, J.; Xiao, J. Evolution of the Rate-Limiting Step: From Thin Film to Thick Ni-Rich Cathodes. J. Power Sources 2020, 454, 227966. https://doi.org/10.1016/j.jpowsour.2020.227966.

(30) Strehle, B.; Friedrich, F.; Gasteiger, H. A. A Comparative Study of Structural Changes during Long-Term Cycling of NCM-811 at Ambient and Elevated Temperatures. J. $\begin{array}{rrrr}\text { Electrochem. Soc. 2021, } & 168, & 050512 .\end{array}$ https://doi.org/10.1149/1945-7111/abf78o.

(31) Li, J.; Downie, L. E.; Ma, L.; Qiu, W.; Dahn, J. R. Study of the Failure Mechanisms of $\mathrm{LiNi}_{0.8} \mathrm{Mn}_{0.1} \mathrm{Co}_{0.1} \mathrm{O}_{2}$ Cathode Material for Lithium Ion Batteries. J. Electrochem. Soc. 2015, 162, A1401-A1408. https://doi.org/10.1149/2.1011507jes.

(32) Märker, K.; Reeves, P. J.; Xu, C.; Griffith, K. J.; Grey, C. P. Evolution of Structure and Lithium Dynamics in $\mathrm{LiNi}_{0.8} \mathrm{Mn}_{0.1} \mathrm{Co}_{0.1} \mathrm{O}_{2} \quad$ (NMC811) Cathodes during Electrochemical Cycling. Chem. Mater. 2019, 31, 2545-2554. https://doi.org/10.1021/acs.chemmater.9boo14o.

(33) Xu, C.; Märker, K.; Lee, J.; Mahadevegowda, A.; Reeves, P. J.; Day, S. J.; Groh, M. F.; Emge, S. P.; Ducati, C.; Layla Mehdi, B.; Tang, C. C.; Grey, C. P. Bulk Fatigue Induced by Surface Reconstruction in Layered Ni-Rich Cathodes for Li-Ion Batteries. Nat. Mater. 2021, 20, 84-92. https://doi.org/10.1038/s41563-020-0767-8.

(34) Xu, C.; Reeves, P. J.; Jacquet, Q.; Grey, C. P. Phase Behavior during Electrochemical Cycling of Ni-Rich Cathode Materials for Li-Ion Batteries. Adv. Energy Mater. 2021, 11, 112. https://doi.org/10.1002/aenm.202003404.

(35) Friedrich, F.; Strehle, B.; Freiberg, A. T. S.; Kleiner, K.; Day, S. J.; Erk, C.; Piana, M.; Gasteiger, H. A. Editors' ChoiceCapacity Fading Mechanisms of NCM-811 Cathodes in Lithium-Ion Batteries Studied by X-Ray Diffraction and Other Diagnostics. J. Electrochem. Soc. 2019, 166, A376oA3774. https://doi.org/10.1149/2.0821915jes. 